\title{
TAIL ASYMPTOTICS OF SIGNAL-TO-INTERFERENCE RATIO DISTRIBUTION IN SPATIAL CELLULAR NETWORK MODELS
}

BY

NAOTO MIYOSHI* (TOKYO) AND TOMOYUKI SHIRAI** (FUKUOKA)

Dedicated to Tomasz Rolski on the occasion of his 70th birthday

\begin{abstract}
We consider a spatial stochastic model of wireless cellular networks, where the base stations (BSs) are deployed according to a simple and stationary point process on $\mathbb{R}^{d}, d \geqslant 2$. In this model, we investigate tail asymptotics of the distribution of signal-to-interference ratio (SIR), which is a key quantity in wireless communications. In the case where the pathloss function representing signal attenuation is unbounded at the origin, we derive the exact tail asymptotics of the SIR distribution under an appropriate sufficient condition. While we show that widely-used models based on a Poisson point process and on a determinantal point process meet the sufficient condition, we also give a counterexample violating it. In the case of bounded path-loss functions, we derive a logarithmically asymptotic upper bound on the SIR tail distribution for the Poisson-based and $\alpha$-Ginibrebased models. A logarithmically asymptotic lower bound with the same order as the upper bound is also obtained for the Poisson-based model.
\end{abstract}

2010 AMS Mathematics Subject Classification: Primary: 60G55; Secondary: 90B18.

Key words and phrases: Spatial point processes, cellular networks, tail asymptotics, signal-to-interference ratio, determinantal point processes.

\section{INTRODUCTION AND MODEL DESCRIPTION}

In this paper, we consider a spatial stochastic model of downlink cellular networks described as follows. Let $\Phi=\left\{X_{i}\right\}_{i \in \mathbb{N}}$ denote a point process on $\mathbb{R}^{d}, d \geqslant 2$ (mostly $d=2$ is supposed), where the points are ordered according to the distance from the origin such that $\left|X_{1}\right| \leqslant\left|X_{2}\right| \leqslant \ldots$ Each point $X_{i}, i \in \mathbb{N}$, represents the location of a base station (BS) of the cellular network, and we refer to

\footnotetext{
* Research supported by JSPS Grant-in-Aid for Scientific Research (C) 16K00030.
}

** Research supported by JSPS Grant-in-Aid for Scientific Research (B) 26287019. 
the BS located at $X_{i}$ as BS $i$. The point process $\Phi$ is assumed to be simple almost surely in probability $\mathrm{P}(\mathrm{P}$-a.s.) and stationary in $\mathrm{P}$ with positive and finite intensity $\lambda=\mathrm{E} \Phi\left([0,1]^{d}\right)$. Assuming further that all the BSs transmit signals at the same power level and each user is associated with the nearest BS, we focus on a typical user located at the origin $o=(0,0, \ldots, 0) \in \mathbb{R}^{d}$. For each $i \in \mathbb{N}$, let $H_{i}$ denote a nonnegative random variable representing the propagation effect of fading and shadowing on the signal from BS $i$ to the typical user, where $H_{i}$, $i \in \mathbb{N}$, are mutually independent and identically distributed (i.i.d.), as well as independent of the point process $\Phi$. The path-loss function representing attenuation of signals with distance is denoted by $\ell$, which is a nonincreasing function satisfying $\int_{\epsilon}^{\infty} r^{d-1} \ell(r) \mathrm{d} r<\infty$ for any $\epsilon>0$. What we have in mind is, for example, $\ell(r)=r^{-d \beta}$ or $\ell(r)=\left(1+r^{d \beta}\right)^{-1}$ with $\beta>1$, the former of which is an example of unbounded path-loss functions, and the latter is bounded.

In this model, the signal-to-interference ratio (SIR) for the typical user is defined as

$$
\mathrm{SIR}_{o}=\frac{H_{1} \ell\left(\left|X_{1}\right|\right)}{\sum_{i=2}^{\infty} H_{i} \ell\left(\left|X_{i}\right|\right)},
$$

where we recall that $X_{1}$ is the nearest point of $\Phi$ from the origin, and the typical user at the origin is associated with BS 1 at $X_{1}$. We can see that $\mathrm{SIR}_{o}$ in (III) is invariant to the intensity $\lambda$ of the point process $\Phi$ when $\ell(r)=r^{-d \beta}$. While SIR is a key quantity in design and analysis of wireless networks, spatial cellular network models where the SIR distribution is obtained exactly in a closed form or a numerically computable form are limited (see, e.g., [1]], [22]). In addition, even when it is numerically computable, the actual computation can be time-consuming (see [22]). Several researchers therefore resort to some approximation and/or asymptotic approaches recently (see, e.g., [5], [12], [14], [16], [19], [23], [25], [27]). In the current paper, we investigate tail asymptotics of the SIR distribution; that is, the asymptotic behavior of $\mathrm{P}\left(\mathrm{SIR}_{o}>\theta\right)$ as $\theta \rightarrow \infty$, for the two cases where the pathloss function is unbounded at the origin and where it is bounded. The part of the unbounded path-loss function is a slight refinement of [25], and we derive the exact tail asymptotics of the SIR distribution under an appropriate sufficient condition. While we show that the widely-used models on $\mathbb{R}^{2}$, where the BS configuration $\Phi$ is given as a homogeneous Poisson point process and where $\Phi$ is a stationary and isotropic determinantal point process, meet the sufficient condition, we also give a counterexample violating it. For the case of bounded path-loss functions, we derive a logarithmically asymptotic upper bound on the SIR tail distribution for the homogeneous Poisson-based and $\alpha$-Ginibre-based models, where the $\alpha$-Ginibre point process is one of the main examples of stationary and isotropic determinantal point processes on $\mathbb{C} \simeq \mathbb{R}^{2}$. We also derive a logarithmically asymptotic lower bound with the same order as the upper bound for the homogeneous Poisson-based model. 
The paper is organized as follows. In the next section, we consider the BS configuration $\Phi$ as a general simple and stationary point process on $\mathbb{R}^{d}$ and the path-loss function $\ell$ as $\ell(r)=r^{-d \beta}, r>0$. In this case, we derive $\mathrm{P}\left(\mathrm{SIR}_{o}>\theta\right)$ $\sim c \theta^{-1 / \beta}$ as $\theta \rightarrow \infty$ for some constant $c \in(0, \infty)$ under an appropriate sufficient condition. In Section B, we show that the widely-used Poisson-based and determinantal-based models on $\mathbb{R}^{2}$ meet the sufficient condition while we also give a counterexample to it. In Section 4 , we consider bounded and regularly varying path-loss functions and derive a logarithmically asymptotic upper bound on $\mathrm{P}\left(\mathrm{SIR}_{o}>\theta\right)$ as $\theta \rightarrow \infty$ when the propagation effect distribution is light-tailed and $\Phi$ is a homogeneous Poisson point process or an $\alpha$-Ginibre point process. When $\Phi$ is a homogeneous Poisson point process and the propagation effects are exponentially distributed, a logarithmically asymptotic lower bound with the same order as the upper bound is also obtained.

\section{TAIL ASYMPTOTICS FOR UNBOUNDED PATH-LOSS MODELS}

In this section, we consider the path-loss function $\ell(r)=r^{-d \beta}, r>0$, and derive $\mathrm{P}\left(\mathrm{SIR}_{o}>\theta\right) \sim c \theta^{-1 / \beta}$ as $\theta \rightarrow \infty$ with some constant $c>0$ under an appropriate set of conditions. Prior to providing the main theorem, we need a short preliminary.

Let $\mathrm{P}^{o}$ and $\mathrm{E}^{o}$ denote respectively the Palm probability and the corresponding expectation with respect to the marked point process $\Phi_{H}=\left\{\left(X_{i}, H_{i}\right)\right\}_{i \in \mathbb{N}}$ viewed at the origin (see, e.g., [3], Section 1.4, or [10], Chapter 13). Note that, due to the independence of $\Phi=\left\{X_{i}\right\}_{i \in \mathbb{N}}$ and $\left\{H_{i}\right\}_{i \in \mathbb{N}}$, we have $\mathrm{P}^{o}\left(H_{1} \in C\right)=$ $\mathrm{P}\left(H_{1} \in C\right)$ for any $C \in \mathcal{B}\left(\mathbb{R}_{+}\right)$. When we consider the point process $\Phi$ under the Palm distribution $\mathrm{P}^{o}$, we use index 0 for the point at the origin; that is, $X_{0}=$ $o=(0,0, \ldots, 0) \in \mathbb{R}^{d}$ under $\mathrm{P}^{o}$. For the point process $\Phi$ and a point $X_{i}$ of $\Phi$, the Voronoi cell of $X_{i}$ with respect to $\Phi$ is defined as

$$
\mathcal{C}\left(X_{i}\right)=\left\{x \in \mathbb{R}^{d}:\left|x-X_{i}\right| \leqslant\left|x-X_{j}\right|, X_{j} \in \Phi\right\} ;
$$

that is, the set of points in $\mathbb{R}^{d}$ whose distance to $X_{i}$ is not greater than that to any other points of $\Phi$. The typical Voronoi cell is then $\mathcal{C}(o)$ under the Palm distribution $\mathrm{P}^{o}$ and its circumscribed radius, denoted by $R(o)$, is the radius of the smallest ball centered at the origin and containing $\mathcal{C}(o)$ under $\mathrm{P}^{o}$.

THEOREM 2.1. For the cellular network model described in the preceding section with the path-loss function $\ell(r)=r^{-d \beta}, r>0$, we suppose the following.

(A) For the propagation effects $H_{i}, i \in \mathbb{N}, \mathrm{E}\left(H_{1}^{1 / \beta}\right)<\infty$ and there exist $p>0$ and $c_{H}>0$ such that the Laplace transform $\mathcal{L}_{H}$ of $H_{i}, i \in \mathbb{N}$, satisfies $\mathcal{L}_{H}(s) \leqslant c_{H} s^{-p}$ for $s \geqslant 1$.

(B) For the point process $\Phi=\left\{X_{i}\right\}_{i \in \mathbb{N}}, \mathrm{E}^{o}\left(R(o)^{d}\right)<\infty$ and there exists a $k>(p \beta)^{-1}$ such that $\mathrm{E}^{o}\left(\left|X_{k}\right|^{d}\right)<\infty$, where $p$ is that in condition (A) above. 
Then we have

$$
\lim _{\theta \rightarrow \infty} \theta^{1 / \beta} \mathrm{P}\left(\mathrm{SIR}_{o}>\theta\right)=\pi_{d} \lambda \mathrm{E}\left(H_{1}{ }^{1 / \beta}\right) \mathrm{E}^{o}\left[\left(\sum_{i=1}^{\infty} \frac{H_{i}}{\left|X_{i}\right|^{d \beta}}\right)^{-1 / \beta}\right],
$$

where $\pi_{d}=\pi^{d / 2} / \Gamma(d / 2+1)$ denotes the volume of a d-dimensional unit ball with the gamma function $\Gamma(x)=\int_{0}^{\infty} t^{x-1} e^{-t} \mathrm{~d} t$.

Theorem 2.1] is a slight extention of [25] to higher dimensions. Recall that $\mathrm{SIR}_{o}$ in (III) is invariant to the intensity $\lambda$ of the point process $\Phi$. Thus, we can show that the right-hand side of (2. I) does not depend on $\lambda$ (see, e.g., the remark of Definition 4 in [12]).

REMARK 2.1. When $d=2$, the right-hand side of (2.J) in Theorem 2.1 coincides with $\mathrm{EFIR}^{\delta}$ in Theorem 4 of [12]; that is, that theorem and our Theorem 2.1$]$ assert the same result. A difference between the two theorems (besides our extention to higher dimensions) is that we offer the set of conditions (A) and (B), the role of which is discussed in the proof and the remarks thereafter.

Proof. Let $F_{H}$ denote the distribution function of $H_{i}, i \in \mathbb{N}$, and let $\overline{F_{H}}(x)$ $=1-F_{H}(x)$. By (II.) with $\ell(r)=r^{-d \beta}, r>0$, the tail probability of the SIR for the typical user is expressed as

$$
\mathrm{P}\left(\mathrm{SIR}_{o}>\theta\right)=\mathrm{E} \overline{F_{H}}\left(\theta\left|X_{1}\right|^{d \beta} \sum_{i=2}^{\infty} \frac{H_{i}}{\left|X_{i}\right|^{d \beta}}\right) .
$$

Applying the Palm inversion formula (see, e.g., [3], Section 4.2) to the right-hand side above, we have

$$
\begin{aligned}
& \mathrm{P}\left(\mathrm{SIR}_{o}>\theta\right) \\
= & \lambda \int_{\mathbb{R}^{d}} \mathrm{E}^{o}\left[\overline{F_{H}}\left(\theta|x|^{d \beta} \sum_{i=1}^{\infty} \frac{H_{i}}{\left|X_{i}-x\right|^{d \beta}}\right) \mathbf{1}_{\mathcal{C}(o)}(x)\right] \mathrm{d} x \\
= & \theta^{-1 / \beta} \lambda \int_{\mathbb{R}^{d}} \mathrm{E}^{o}\left[\overline{F_{H}}\left(|y|^{d \beta} \sum_{i=1}^{\infty} \frac{H_{i}}{\left|X_{i}-\theta^{-1 /(d \beta)} y\right|^{d \beta}}\right) \mathbf{1}_{\mathcal{C}(o)}\left(\theta^{-1 /(d \beta)} y\right)\right] \mathrm{d} y,
\end{aligned}
$$

where the second equality follows from the substitution of $y=\theta^{1 /(d \beta)} x$. Therefore, if we can find a random function $A$ satisfying

$$
\begin{gathered}
\overline{F_{H}}\left(|y|^{d \beta} \sum_{i=1}^{\infty} \frac{H_{i}}{\left|X_{i}-\theta^{-1 /(d \beta)} y\right|^{d \beta}}\right) \mathbf{1}_{\mathcal{C}(o)}\left(\theta^{-1 /(d \beta)} y\right) \leqslant A(y), \quad \mathrm{P}^{o} \text {-a.s. } \\
\int_{\mathbb{R}^{d}} \mathrm{E}^{o} A(y) \mathrm{d} y<\infty
\end{gathered}
$$


the dominated convergence theorem yields

$$
\lim _{\theta \rightarrow \infty} \theta^{1 / \beta} \mathrm{P}\left(\mathrm{SIR}_{o}>\theta\right)=\lambda \int_{\mathbb{R}^{d}} \mathrm{E}^{o} \overline{F_{H}}\left(|y|^{d \beta} \sum_{i=1}^{\infty} \frac{H_{i}}{\left|X_{i}\right|^{\mid \beta}}\right) \mathrm{d} y .
$$

We postpone finding such a function $A$ and admit (2.5) for a moment. Then, substituting $z=\left(\sum_{i=1}^{\infty} H_{i} /\left|X_{i}\right|^{d \beta}\right)^{1 /(d \beta)} y$ to the integral in (2.5)), we have

$$
\int_{\mathbb{R}^{d}} \mathrm{E}^{o} \overline{F_{H}}\left(|y|^{d \beta} \sum_{i=1}^{\infty} \frac{H_{i}}{\left|X_{i}\right|^{d \beta}}\right) \mathrm{d} y=\mathrm{E}^{o}\left[\left(\sum_{i=1}^{\infty} \frac{H_{i}}{\left|X_{i}\right|^{d \beta}}\right)^{-1 / \beta}\right] \int_{\mathbb{R}^{d}} \overline{F_{H}}\left(|z|^{d \beta}\right) \mathrm{d} z,
$$

and the integral on the right-hand side above further reduces to

$$
\begin{aligned}
\int_{\mathbb{R}^{d}} \overline{F_{H}}\left(|z|^{d \beta}\right) \mathrm{d} z & =d \pi_{d} \int_{0}^{\infty} \overline{F_{H}}\left(r^{d \beta}\right) r^{d-1} \mathrm{~d} r \\
& =\frac{\pi_{d}}{\beta} \int_{0}^{\infty} \overline{F_{H}}(s) s^{-1+1 / \beta} \mathrm{d} s=\pi_{d} \mathrm{E}\left(H_{1}{ }^{1 / \beta}\right) .
\end{aligned}
$$

Hence, applying ([2.6) and (2.7) to (2.5)), we obtain ([2.1).

It remains to find a function $A$ satisfying (2.3) and (2.4). Since $\overline{F_{H}}$ is nonincreasing and $\left|X_{i}-y\right| \leqslant\left|X_{i}\right|+R(o) \mathrm{P}^{o}$-a.s. for $y \in \mathcal{C}(o)$, we can set a function $A$ satisfying (2.3) as

$$
A(y)=\overline{F_{H}}\left(|y|^{d \beta} \sum_{i=1}^{\infty} \frac{H_{i}}{\left(\left|X_{i}\right|+R(o)\right)^{d \beta}}\right) .
$$

We now confirm that this function $A$ satisfies condition (2.4). By substituting $z=$ $\left(\sum_{i=1}^{\infty} H_{i} /\left(\left|X_{i}\right|+R(o)\right)^{d \beta}\right)^{1 /(d \beta)} y$ and using (2.7) again, we have

$$
\int_{\mathbb{R}^{d}} \mathrm{E}^{o} A(y) \mathrm{d} y=\pi_{d} \mathrm{E}\left(H_{1}{ }^{1 / \beta}\right) \mathrm{E}^{o}\left[\left(\sum_{i=1}^{\infty} \frac{H_{i}}{\left(\left|X_{i}\right|+R(o)\right)^{d \beta}}\right)^{-1 / \beta}\right],
$$

where $\mathrm{E}\left(H_{1}^{1 / \beta}\right)<\infty$ by condition (A). Applying the identity

$$
x^{-1 / \beta}=\frac{1}{\Gamma(1 / \beta)} \int_{0}^{\infty} e^{-x s} s^{-1+1 / \beta} \mathrm{d} s
$$

to the second expectation on the right-hand side of $(2.8)$, we have

$$
\begin{aligned}
\mathrm{E}^{o}\left[\left(\sum_{i=1}^{\infty} \frac{H_{i}}{\left(\left|X_{i}\right|+R(o)\right)^{d \beta}}\right)^{-1 / \beta}\right] \\
=\frac{1}{\Gamma(1 / \beta)} \int_{0}^{\infty} s^{-1+1 / \beta} \mathrm{E}^{o}\left[\prod_{i=1}^{\infty} \mathcal{L}_{H}\left(\frac{s}{\left(\left|X_{i}\right|+R(o)\right)^{d \beta}}\right)\right] \mathrm{d} s .
\end{aligned}
$$


Recall that $X_{i}, i \in \mathbb{N}$, are ordered such that $\left|X_{1}\right|<\left|X_{2}\right|<\ldots$ By truncating the infinite product above by a finite $k \in \mathbb{N}$ such that $p \beta k>1$ and applying $\mathcal{L}_{H}(s) \leqslant$ $c_{H} s^{-p}$ for $s \geqslant 1$ from condition (A), we can bound the integral on the right-hand side above by

$$
\begin{aligned}
\int_{0}^{\infty} s^{-1+1 / \beta} & \mathrm{E}^{o}\left[\prod_{i=1}^{k} \mathcal{L}_{H}\left(\frac{s}{\left(\left|X_{i}\right|+R(o)\right)^{d \beta}}\right)\right] \mathrm{d} s \\
& \leqslant \int_{0}^{\infty} s^{-1+1 / \beta} \mathrm{E}^{o}\left[\left\{\mathcal{L}_{H}\left(\frac{s}{\left(\left|X_{k}\right|+R(o)\right)^{d \beta}}\right)\right\}^{k}\right] \mathrm{d} s \\
& \leqslant \mathrm{E}^{o}\left[\int_{0}^{\left(\left|X_{k}\right|+R(o)\right)^{d \beta}} s^{-1+1 / \beta} \mathrm{d} s\right] \\
& +c_{H}{ }^{k} \mathrm{E}^{o}\left[\left(\left|X_{k}\right|+R(o)\right)^{d p \beta k} \int_{\left(\left|X_{k}\right|+R(o)\right)^{d \beta}}^{\infty} s^{-1+1 / \beta-p k} \mathrm{~d} s\right] \\
& =\beta\left(1+\frac{c_{H}{ }^{k}}{p \beta k-1}\right) \mathrm{E}^{o}\left[\left(\left|X_{k}\right|+R(o)\right)^{d}\right] .
\end{aligned}
$$

Hence, the inequality $(a+b)^{d} \leqslant 2^{d-1}\left(a^{d}+b^{d}\right)$ ensures (2.4) under condition (B) of the theorem.

REMARK 2.2. The differences between the proof in [12] and ours are as follows. The first and less essential one is that, in [12], they arrange the right-hand side of (2.2) into a certain appropriate form and then apply the Campbell-Mecke formula (see, e.g., [3], Section 1.4). On the other hand, we apply the Palm inversion formula directly. Second, [12] does not specify any condition under which the result holds. However, equality ([.5) requires some kind of uniform integrability condition to change the order of the limit and integrals. Our set of conditions (A) and (B) gives a sufficient condition for this order change to be valid and complements the proof of [112].

REMARK 2.3. Condition (A) claims that the Laplace transform of $H_{i}, i \in \mathbb{N}$, decays faster than or equal to power laws. Though this condition excludes distributions with a mass at the origin, it covers many practical distributions. For example, gamma distribution $\operatorname{Gam}(p, q), p>0, q>0$, has the Laplace transform $\mathcal{L}_{H}(s)=(1+q s)^{-p}$, and we can take $c_{H} \geqslant q^{-p}$. In addition, we can see from the results of [2] that lognormal distributions also satisfy condition (A).

The asymptotic constant in (2.1) of Theorem [2.] depends on the point process $\Phi$ and the distribution $F_{H}$ of the propagation effects. The following proposition indicates an impact of the propagation effect distribution on the asymptotic constant by comparing with the case without propagation effects. 
PROPOSITION 2.1. Let $C\left(\beta, F_{H}\right)$ denote the asymptotic constant on the righthand side of (․]I), specifying the dependence on $\beta$ and the distribution $F_{H}$ of propagation effects. When $\mathrm{E} H_{1}<\infty$, we have

$$
C\left(\beta, F_{H}\right) \geqslant \frac{\mathrm{E}\left(H_{1}^{1 / \beta}\right)}{\left(\mathrm{E} H_{1}\right)^{1 / \beta}} C\left(\beta, \delta_{1}\right),
$$

where $\delta_{1}$ denotes the Dirac measure with mass at one.

P r o o f. The result immediately follows from Jensen's inequality conditioned on $\Phi=\left\{X_{i}\right\}_{i \in \mathbb{N}}$. On the right-hand side of (2.1), since $f(x)=x^{-1 / \beta}$ is convex for $x>0$,

$$
\begin{aligned}
\mathrm{E}^{o}\left[\left(\sum_{i \in \mathbb{N}} \frac{H_{i}}{\left|X_{i}\right|^{d \beta}}\right)^{-1 / \beta}\right] & \geqslant \mathrm{E}^{o}\left[\left(\sum_{i \in \mathbb{N}} \frac{\mathrm{E} H_{i}}{\left|X_{i}\right|^{d \beta}}\right)^{-1 / \beta}\right] \\
& =\frac{1}{\left(\mathrm{E} H_{1}\right)^{1 / \beta}} \mathrm{E}^{o}\left[\left(\sum_{i \in \mathbb{N}} \frac{1}{\left|X_{i}\right|^{d \beta}}\right)^{-1 / \beta}\right],
\end{aligned}
$$

and (2.9) holds.

REMARK 2.4. When $F_{H}=\operatorname{Exp}(1)$, denoting the exponential distribution with unit mean (which assumes Rayleigh fading and ignores shadowing), the result of Proposition 2.] reduces to the second part of Theorem 2 in [24] since $\mathrm{E}\left(H_{1}^{1 / \beta}\right)=\Gamma(1+1 / \beta)$ in this case (though only the Ginibre point process $\Phi$ is considered there). In inequality (2.9), it is easy to see (by Jensen's inequality for a concave function $\left.f(x)=x^{1 / \beta}\right)$ that the coefficient $\mathrm{E}\left(H_{1}^{1 / \beta}\right) /\left(\mathrm{E} H_{1}\right)^{1 / \beta}$ is not greater than one. Now, suppose that $\mathrm{E} H_{1}=1$. Then, the dominated convergence theorem (due to $H_{1}^{1 / \beta} \leqslant 1+H_{1}$ a.s.) leads to $\mathrm{E}\left(H_{1}^{1 / \beta}\right) \rightarrow 1$ as both $\beta \downarrow 1$ and $\beta \uparrow \infty$, which implies that $C\left(\beta, F_{H}\right)$ tends to be larger than or equal to $C\left(\beta, \delta_{1}\right)$ when $\beta$ is close to one or sufficiently large.

\section{EXAMPLES FOR UNBOUNDED PATH-LOSS MODELS}

In this section, we restrict ourselves to the case of $d=2$ and provide a few examples demonstrating Theorem 2.1 of the preceding section. We also give a counterexample violating condition (B) of the theorem.

3.1. Poisson-based model. We consider here the BS configuration $\Phi$ as a homogeneous Poisson point process on $\mathbb{R}^{2}$ with positive and finite intensity. We first confirm that $\Phi$ satisfies condition (B) of Theorem 2.1. 
LEMMA 3.1. Let $\Phi=\left\{X_{i}\right\}_{i \in \mathbb{N}}$ denote a homogeneous Poisson point process on $\mathbb{R}^{2}$ with positive and finite intensity. Then, for $\epsilon>0$,

$$
\begin{aligned}
& \mathrm{E}^{o} e^{\epsilon\left|X_{k}\right|}<\infty, \quad k \in \mathbb{N}, \\
& \mathrm{E}^{o} e^{\epsilon R(o)}<\infty .
\end{aligned}
$$

This lemma ensures that $\left|X_{k}\right|, k \in \mathbb{N}$, and $R(o)$ have any order of moments.

Pro of. Let $\lambda$ denote the intensity of $\Phi$ and let $D_{r}$ denote the disk centered at the origin with radius $r>0$. Recalling that $X_{i}, i \in \mathbb{N}$, are ordered such that $\left|X_{1}\right|<\left|X_{2}\right|<\ldots$, we have

$$
\mathrm{P}^{o}\left(\left|X_{k}\right|>r\right)=\mathrm{P}\left(\left|X_{k}\right|>r\right)=\mathrm{P}\left(\Phi\left(D_{r}\right)<k\right)=e^{-\lambda \pi r^{2}} \sum_{j=0}^{k-1} \frac{\left(\lambda \pi r^{2}\right)^{j}}{j !} .
$$

Thus, we can use the density function of $\left|X_{k}\right|$ and show (B.T).

On the other hand, for the circumscribed radius $R(o)$ of the typical Voronoi cell of $\Phi$, Calka [8], Theorem 3, shows that there exists an $r_{0} \in(0, \infty)$ such that

$$
\mathrm{P}^{o}(R(o)>r) \leqslant 4 \pi \lambda r^{2} e^{-\pi \lambda r^{2}} \quad \text { for } r \geqslant r_{0},
$$

and we can show (B.2) by applying $\mathrm{E}^{o} e^{\epsilon R(o)}=1+\epsilon \int_{0}^{\infty} e^{\epsilon r} \mathrm{P}^{o}(R(o)>r) \mathrm{d} r$.

Now, we apply Theorem 2.$]$ and obtain the following.

COROLlaRY 3.1. Suppose that $\Phi=\left\{X_{i}\right\}_{i \in \mathbb{N}}$ is a homogeneous Poisson point process on $\mathbb{R}^{2}$. When the propagation effects $H_{i}, i \in \mathbb{N}$, satisfy condition (A) of Theorem $\mathbb{2 . 1}$, the right-hand side of (‥]) reduces to $(\beta / \pi) \sin (\pi / \beta)$.

Pr o of. Since the conditions of Theorem 2.1 are fulfilled, the result follows from the proof of Lemma 6 in [12].

REMARK 3.1. The asymptotic result in Corollary B.] agrees with that in Remark 4 of [22], where only Rayleigh fading is considered. Corollary B.1] states that the SIR tail probability in the homogeneous Poisson-based model is asymptotically insensitive to the distribution of propagation effects as far as it satisfies condition (A) of Theorem 2.].

3.2. Determinantal-based model. In this subsection, we consider $\Phi$ as a general stationary and isotropic determinantal point process on $\mathbb{C} \simeq \mathbb{R}^{2}$ with intensity $\lambda$. Let $K: \mathbb{C}^{2} \rightarrow \mathbb{C}$ denote the kernel of $\Phi$ with respect to the Lebesgue measure. The product density functions (joint intensities) $\rho_{n}, n \in \mathbb{N}$, with respect to the Lebesgue measure are given by

$$
\rho_{n}\left(z_{1}, z_{2}, \ldots, z_{n}\right)=\operatorname{det}\left(K\left(z_{i}, z_{j}\right)\right)_{i, j=1,2, \ldots, n} \text { for } z_{1}, z_{2}, \ldots, z_{n} \in \mathbb{C},
$$


where det denotes the determinant. In order for the point process $\Phi$ to be welldefined, we assume that (i) the kernel $K$ is continuous on $\mathbb{C} \times \mathbb{C}$; (ii) $K$ is Hermitian in the sense that $K(z, w)=\overline{K(w, z)}$ for $z, w \in \mathbb{C}$, where $\bar{z}$ denotes the complex conjugate of $z \in \mathbb{C}$; and (iii) the integral operator on $L^{2}(\mathbb{C})$ corresponding to $K$ is of locally trace class with the spectrum in $[0,1]$; that is, for a compact set $C \in \mathcal{B}(\mathbb{C})$, the restriction $K_{C}$ of $K$ on $C$ has the eigenvalues $\kappa_{C, i}, i \in \mathbb{N}$, satisfying $\sum_{i \in \mathbb{N}} \kappa_{C, i}<\infty$ and $\kappa_{C, i} \in[0,1]$ for each $i \in \mathbb{N}$ (see, e.g., [118], Chapter 4). Furthermore, for stationarity and isotropy, the kernel $K$ is assumed to satisfy $|K(z, w)|^{2}=|K(0, z-w)|^{2}$ which depends only on the distance $|z-w|$ of $z$ and $w \in \mathbb{C}$. The product density functions $\rho_{n}, n \in \mathbb{N}$, are then motion-invariant (invariant to translations and rotations), and we infer that $\rho_{1}(z)=K(z, z)=\lambda$ and that $\rho_{2}(0, z)=\lambda^{2}-|K(0, z)|^{2}$ depends only on $|z|$ for $z \in \mathbb{C}$. An $\alpha$-Ginibre point process with $\alpha \in(0,1]$ is one of the main examples of stationary and isotropic determinantal point processes on $\mathbb{C}$, and its kernel is given by

$$
K_{\alpha}(z, w)=\frac{1}{\pi} e^{-\left(|z|^{2}+|w|^{2}\right) /(2 \alpha)} e^{z \bar{w} / \alpha}, \quad z, w \in \mathbb{C},
$$

with respect to the Lebesgue measure (see, e.g., [13], [26]). We can see that the intensity and the second product density of the $\alpha$-Ginibre point process are $\lambda=$ $\rho_{1}^{(\alpha)}(0)=\pi^{-1}$ and $\rho_{2}^{(\alpha)}(0, z)=\left(1-e^{-|z|^{2} / \alpha}\right) / \pi^{2}$, respectively.

First, concerning condition (B) of Theorem 2.1 , we show the following.

LEMMA 3.2. Let $\Phi$ denote a stationary and isotropic determinantal point process on $\mathbb{C}$ with positive and finite intensity as described above.

(i) Let $X_{i}, i \in \mathbb{N}$, denote the points of $\Phi$ such that $\left|X_{1}\right|<\left|X_{2}\right|<\ldots$ Then, there exist $a_{1}>0$ and $a_{2}>0$ such that, for any $k \in \mathbb{N}$, we can take an $r_{k}>0$ satisfying

$$
\mathrm{P}^{o}\left(\left|X_{k}\right|>r\right) \leqslant a_{1} e^{-a_{2} r^{2}} \quad \text { for } r \geqslant r_{k} .
$$

(ii) Let $R(o)$ denote the circumscribed radius of the typical Voronoi cell $\mathcal{C}(o)$ of $\Phi$. Then, there exist $b_{1}>0$ and $b_{2}>0$ such that

$$
\mathrm{P}^{o}(R(o)>r) \leqslant b_{1} e^{-b_{2} r^{2}} \quad \text { for } r>0 .
$$

By Lemma 3.2, it is easy to confirm, as in Lemma [3.], that $\left|X_{k}\right|, k \in \mathbb{N}$, and $R(o)$ have any order of moments under $\mathrm{P}^{o}$. To prove Lemma 3.2, we use the following supplementary lemma.

LEMMA 3.3. The kernel $K$ of a determinantal point process $\Phi$ satisfies

$$
\int_{\mathbb{C}}|K(z, w)|^{2} \mathrm{~d} w \leqslant K(z, z), \quad z \in \mathbb{C} .
$$

Pro of. For a compact set $C \in \mathcal{B}(\mathbb{C})$ such that $z \in C$, let $K_{C}$ denote the restriction of $K$ on $C$. Let also $\kappa_{C, i}$ and $\varphi_{C, i}, i \in \mathbb{N}$, denote, respectively, the 
nonzero eigenvalues of $K_{C}$ and the corresponding orthonormal eigenfunctions; that is,

$$
\int_{C} \varphi_{C, i}(z) \overline{\varphi_{C, j}(z)} \mathrm{d} z= \begin{cases}1 & \text { for } i=j, \\ 0 & \text { for } i \neq j .\end{cases}
$$

Then Mercer's theorem states that the following spectral expansion holds (see, e.g., [2T]):

$$
K_{C}(z, w)=\sum_{i=1}^{\infty} \kappa_{C, i} \varphi_{C, i}(z) \overline{\varphi_{C, i}(w)}, \quad z, w \in C .
$$

Thus, we have

$$
\begin{aligned}
\int_{C}|K(z, w)|^{2} \mathrm{~d} w & =\int_{C}\left|K_{C}(z, w)\right|^{2} \mathrm{~d} w=\sum_{i=1}^{\infty} \kappa_{C, i}{ }^{2}\left|\varphi_{C, i}(z)\right|^{2} \\
& \leqslant K_{C}(z, z)=K(z, z),
\end{aligned}
$$

where the second equality follows from (3.7) and (3.8), the inequality holds since $\kappa_{C, i} \in(0,1], i \in \mathbb{N}$, and the last equality follows since $z \in C$. Finally, by letting $C \uparrow \mathbb{C}$, we obtain (3.6).

Note that Lemma 3.3 implies that $\int_{\mathbb{C}}|K(0, z)|^{2} \mathrm{~d} z \leqslant \lambda$ in our stationary case with intensity $\lambda \in(0, \infty)$. Using this, we prove Lemma 3.2 as follows.

Pro of of Le m m a 3.2. Let $\mathrm{P}^{!}$denote the reduced Palm probability with respect to the marked point process $\Phi_{H}=\left\{\left(X_{i}, H_{i}\right)\right\}_{i \in \mathbb{N}}$, and let $C$ denote a bounded set in $\mathcal{B}(\mathbb{C})$. Since a determinantal point process is also determinantal under the (reduced) Palm distribution (see, e.g., [29]), $\Phi(C)$ under $\mathrm{P}$ ! has the same distribution as $\sum_{i \in \mathbb{N}} B_{C, i}$ with certain mutually independent Bernoulli random variables $B_{C, i}, i \in \mathbb{N}$ (see, e.g., [18], Section 4.5). Thus, the Chernoff-Hoeffding bound for an infinite sum with finite mean (see, e.g., [9], [17] for a finite sum) states that, for any $\epsilon \in[0,1)$, there exists a $c_{\epsilon}>0$ such that

$$
\mathrm{P}^{!}\left(\Phi(C) \leqslant \epsilon \mathrm{E}^{!} \Phi(C)\right) \leqslant e^{-c_{\epsilon} \mathrm{E}^{!} \Phi(C)},
$$

where $E^{!}$denotes the expectation with respect to $P !$. On the other hand, the kernel of $\Phi$ under the reduced Palm distribution is given by (see [29])

$$
K^{!}(z, w)=\frac{K(z, w) K(0,0)-K(z, 0) K(0, w)}{K(0,0)}, \quad z, w \in \mathbb{C},
$$

whenever $K(0,0)>0$, which is ensured in our stationary case with $K(0,0)=\lambda$. Therefore, the intensity function of $\Phi$ under $P$ ! reduces to

$$
\rho_{1}^{!}(z)=K^{!}(z, z)=\lambda-\frac{|K(0, z)|^{2}}{\lambda},
$$


so that Lemma 3.3 with $K(0,0)=\lambda$ yields

$$
\mathrm{E}^{!} \Phi(C)=\int_{C} \rho_{1}^{!}(z) \mathrm{d} z \geqslant \lambda \mu(C)-1,
$$

where $\mu$ denotes the Lebesgue measure on $\mathbb{C}$.

Pr o of of (i). Note that $\mathrm{P}^{o}\left(\left|X_{k}\right|>r\right)=\mathrm{P}^{!}\left(\Phi\left(D_{r}\right) \leqslant k-1\right)$. Since we have $\mathrm{E}^{!} \Phi\left(D_{r}\right) \geqslant \lambda \pi r^{2}-1$ from (B.D), applying this to (B.9) yields

$$
\mathrm{P}^{!}\left(\Phi\left(D_{r}\right) \leqslant \epsilon\left(\lambda \pi r^{2}-1\right)\right) \leqslant e^{c_{\epsilon}} e^{-c_{\epsilon} \lambda \pi r^{2}} .
$$

Hence, for any $\epsilon \in(0,1)$ and $k \in \mathbb{N}$, we can take $r_{k}>0$ satisfying $\epsilon\left(\lambda \pi r_{k}^{2}-1\right)$ $\geqslant k-1$, which implies (B.4).

Pr o of of (ii). We derive here an upper bound on $\mathrm{P}^{o}(R(o)>r)$ by exploiting Foss \& Zuyev's seven petals [1]], which are considered to obtain an upper bound on the tail distribution of the circumscribed radius of the typical PoissonVoronoi cell. Consider a collection of seven disks with a common radius $r$ centered at points $(r, 2 \pi k / 7), k=0,1, \ldots, 6$, in polar coordinates. The petal 0 is given as the intersection of the two disks centered at $(r, 0),(r, 2 \pi / 7)$ and the angular domain between the rays $\phi=0$ and $\phi=2 \pi / 7$. The petal $k$ is the rotation copy of petal 0 by angle $2 \pi k / 7$ for $k=1,2, \ldots, 6$ (see Figure $\mathbb{\text { I) }}$. Let $\mathcal{P}_{r, k}, k=0,1, \ldots, 6$, denote the set formed by petal $k$ on the complex plane $\mathbb{C}$. Then, according to the discussion in the proof of Lemma 1 of [П]],

$$
\mathrm{P}^{o}(R(o)>r) \leqslant \mathrm{P}^{!}\left(\bigcup_{k=0}^{6}\left\{\Phi\left(\mathcal{P}_{r, k}\right)=0\right\}\right) \leqslant 7 \mathrm{P}^{!}\left(\Phi\left(\mathcal{P}_{r, 0}\right)=0\right),
$$

where the second inequality follows from the isotropy of $\Phi$ under the Palm distribution. Now, we can apply inequality (B.9) with $\epsilon=0$, and we have

$$
\mathrm{P}^{!}\left(\Phi\left(\mathcal{P}_{r, 0}\right)=0\right) \leqslant e^{-c_{0} \mathrm{E}^{!} \Phi\left(\mathcal{P}_{r, 0}\right)} .
$$

Hence, (B.5) follows from $\mathrm{E}^{!} \Phi\left(\mathcal{P}_{r, 0}\right) \geqslant \lambda \mu\left(\mathcal{P}_{r, 0}\right)-1$ and $\mu\left(\mathcal{P}_{r, 0}\right)=2 r^{2}(\pi / 7+$ $\sin (\pi / 7) \cos (3 \pi / 7))$.

REMARK 3.2. The first part (i) of Lemma 3.2 (as well as the first part (B.D) of Lemma B. I) can be extended to a determinantal point process on $\mathbb{R}^{d}$ (see [7], Lemma 5.6). We can take $c_{0}$ in (3.13) equal to one since determinantal point processes are weakly sub-Poisson (in particular, due to the $\nu$-weakly sub-Poisson property) (see [6] for details).

REMARK 3.3. When the kernel $K$ of a determinantal point process is explicitly specified, it may be possible to obtain a tighter upper bound on the tail probability of the circumscribed radius of the typical Voronoi cell. For example, the case of an $\alpha$-Ginibre point process is given by the following corollary. 


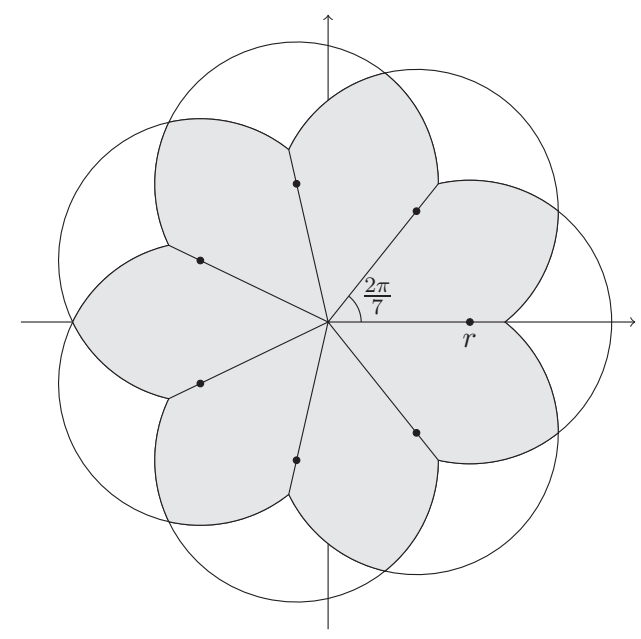

Figure 1. Foss \& Zuyev's seven petals (see [1]]).

COROLLARY 3.2. For an $\alpha$-Ginibre point process, the circumscribed radius for the typical Voronoi cell $\mathcal{C}(o)$ satisfies

$$
\mathrm{P}^{o}(R(o)>r) \leqslant 7 e^{-\left(u_{\alpha}(r) \vee v_{\alpha}(r)\right)} \quad \text { for } r>0,
$$

where $a \vee b=\max (a, b)$ and

$$
\begin{aligned}
& u_{\alpha}(r)=\frac{1}{7}\left\{4 r^{2} \cos ^{2} \frac{2 \pi}{7}-\alpha\left[1-\exp \left(-\frac{4 r^{2}}{\alpha} \cos ^{2} \frac{2 \pi}{7}\right)\right]\right\}, \\
& v_{\alpha}(r)=\frac{2 r^{2}}{\pi}\left(\frac{\pi}{7}+\sin \frac{\pi}{7} \cos \frac{3 \pi}{7}\right)-\frac{\alpha}{7}\left[1-\exp \left(-\frac{4 r^{2}}{\alpha} \cos ^{2} \frac{\pi}{7}\right)\right] .
\end{aligned}
$$

Pro of. By the kernel of the $\alpha$-Ginibre point process in (B.3), the intensity function of (B.10) under the (reduced) Palm distribution reduces to

$$
\rho_{1}^{!}(z)=\frac{1}{\pi}\left(1-e^{-|z|^{2} / \alpha}\right), \quad z \in \mathbb{C} .
$$

We obtain two lower bounds of $\mathrm{E}^{!} \Phi\left(\mathcal{P}_{r, 0}\right)$ as follows. Let $\mathcal{S}_{\eta}$ denote the circular sector centered at the origin with radius $\eta$ and the angular domain between $\phi=0$ and $\phi=2 \pi / 7$. Taking $\eta_{1}=2 r \cos (2 \pi / 7)$ and $\eta_{2}=2 r \cos (\pi / 7)$, we have $\mathcal{S}_{\eta_{1}} \subset$ $\mathcal{P}_{r, 0} \subset \mathcal{S}_{\eta_{2}}$. Therefore, applying (B.15), we have the first lower bound:

$$
\mathrm{E}^{!} \Phi\left(\mathcal{P}_{r, 0}\right) \geqslant \mathrm{E}^{!} \Phi\left(\mathcal{S}_{\eta_{1}}\right)=\int_{\mathcal{S}_{\eta_{1}}} \rho_{1}^{!}(z) \mathrm{d} z=\frac{1}{7}\left[\eta_{1}{ }^{2}+\alpha\left(e^{-\eta_{1}{ }^{2} / \alpha}-1\right)\right]=u_{\alpha}(r) .
$$

The second lower bound is given by

$$
\mathrm{E}^{!} \Phi\left(\mathcal{P}_{r, 0}\right)=\int_{\mathcal{P}_{r, 0}} \rho_{1}^{!}(z) \mathrm{d} z \geqslant \frac{1}{\pi}\left(\mu\left(\mathcal{P}_{r, 0}\right)-\int_{\mathcal{S}_{\eta_{2}}} e^{-|z|^{2} / \alpha} \mathrm{d} z\right)=v_{\alpha}(r) .
$$

Hence, we have (B.14) from (3.T2) and (B.T3) with $c_{0}=1$. 
Indeed, when, e.g., $\alpha=1$, we can numerically compute $r_{*} \approx 0.5276 \ldots$ such that $u_{1}(r)>v_{1}(r)$ for $r<r_{*}$ and $u_{1}(r)<v_{1}(r)$ for $r>r_{*}$. We are now ready to give the tail asymptotics of the SIR distribution when the BSs are deployed according to an $\alpha$-Ginibre point process.

COROLlaRY 3.3. Suppose that $\Phi=\left\{X_{i}\right\}_{i \in \mathbb{N}}$ is an $\alpha$-Ginibre point process. When the propagation effects $H_{i}, i \in \mathbb{N}$, satisfy condition (A) of Theorem $\mathbb{2 . 1}$, we have

$$
\begin{aligned}
& \lim _{\theta \rightarrow \infty} \theta^{1 / \beta} \mathrm{P}\left(\mathrm{SIR}_{o}>\theta\right) \\
& \quad=\frac{\alpha \mathrm{E}\left(H_{1}{ }^{1 / \beta}\right)}{\Gamma(1+1 / \beta)} \int_{0}^{\infty} \prod_{i=1}^{\infty}\left[1-\alpha+\frac{\alpha}{i !} \int_{0}^{\infty} e^{-y} y^{i} \mathcal{L}_{H}\left(\left(\frac{t}{y}\right)^{\beta}\right) \mathrm{d} y\right] \mathrm{d} t .
\end{aligned}
$$

For the proof of Corollary B.3, we use the following proposition which is a consequence of [13] and [20] (see also [26]).

Proposition 3.1. (i) Let $X_{i}, i \in \mathbb{N}$, denote the points of an $\alpha$-Ginibre point process. Then, the set $\left\{\left|X_{i}\right|^{2}\right\}_{i \in \mathbb{N}}$ has the same distribution as $\tilde{\boldsymbol{Y}}=\left\{\check{Y}_{i}\right\}_{i \in \mathbb{N}}$, which is extracted from $\boldsymbol{Y}=\left\{Y_{i}\right\}_{i \in \mathbb{N}}$ such that $Y_{i}, i \in \mathbb{N}$, are mutually independent with $Y_{i} \sim \operatorname{Gam}(i, \alpha)$ for each $i \in \mathbb{N}$, and each $Y_{i}$ is added in $\check{\boldsymbol{Y}}$ with probability $\alpha$ and discarded with $1-\alpha$ independently of others.

(ii) Let $X_{i}, i \in \mathbb{N}$, denote the points of an $\alpha$-Ginibre point process under the reduced Palm distribution. Then, the same statement as (i) holds except for replacing $Y_{i} \sim \operatorname{Gam}(i, \alpha)$ by $Y_{i} \sim \operatorname{Gam}(i+1, \alpha)$.

Pro of of Corollary 3.3 . For an $\alpha$-Ginibre point process, we can see by Lemma 3.2 (or Corollary [3.2) that $\left|X_{k}\right|, k \in \mathbb{N}$, and $R(o)$ have any order of moments under the Palm distribution $\mathrm{P}^{o}$; that is, condition (B) of Theorem $2 . \mathrm{C}$ is fulfilled. Thus, applying the identity $x^{-1 / \beta}=\Gamma(1 / \beta)^{-1} \int_{0}^{\infty} e^{-x s} s^{-1+1 / \beta} \mathrm{d} s$ and the Laplace transform $\mathcal{L}_{H}$ to the right-hand side of (2.1), we have

$$
\begin{aligned}
\mathrm{E}^{o}\left[\left(\sum_{i=1}^{\infty} \frac{H_{i}}{\left|X_{i}\right|^{2 \beta}}\right)^{-1 / \beta}\right] & =\frac{1}{\Gamma(1 / \beta)} \int_{0}^{\infty} s^{-1+1 / \beta} \mathrm{E}^{o}\left[\prod_{i=1}^{\infty} \mathcal{L}_{H}\left(\frac{s}{\left|X_{i}\right|^{2 \beta}}\right)\right] \mathrm{d} s \\
& =\frac{1}{\Gamma(1+1 / \beta)} \int_{0}^{\infty} \mathrm{E}^{o}\left[\prod_{i=1}^{\infty} \mathcal{L}_{H}\left(\left(\frac{t}{\left|X_{i}\right|^{2}}\right)^{\beta}\right)\right] \mathrm{d} t
\end{aligned}
$$

where the second equality follows by substituting $t=s^{1 / \beta}$. Here, applying $\boldsymbol{Y}=$ $\left\{Y_{i}\right\}_{i \in \mathbb{N}}$ in Proposition $3 . \mathbb{J}$ (ii), we have (B.J6).

REMARK 3.4. When $F_{H}=\operatorname{Exp}(1)$ (Rayleigh fading without shadowing), (13.16) reduces to the result of Theorem 1 in [23]. When $F_{H}=\operatorname{Gam}(m, 1 / m)$ (Nakagami-m fading without shadowing), we have $\mathcal{L}_{H}(s)=(1+s / m)^{-m}$ and 
$\mathrm{E}\left(H_{1}{ }^{1 / \beta}\right)=\Gamma(m+1 / \beta) /\left(m^{1 / \beta}(m-1) !\right)$. Applying these to the right-hand side of (B.16) yields

$$
\begin{aligned}
& \lim _{\theta \rightarrow \infty} \theta^{1 / \beta} \mathrm{P}\left(\mathrm{SIR}_{o}>\theta\right) \\
= & \frac{\alpha \Gamma(m+1 / \beta)}{\Gamma(1+1 / \beta) m^{1 / \beta}(m-1) !} \int_{0}^{\infty} \prod_{i=1}^{\infty}\left[1-\alpha+\frac{\alpha}{i !} \int_{0}^{\infty} \frac{e^{-y} y^{i}}{\left(1+m^{-1}(t / y)^{\beta}\right)^{m}} \mathrm{~d} y\right] \mathrm{d} t \\
= & \frac{\alpha \beta}{B(m, 1 / \beta)} \int_{0}^{\infty} \prod_{i=1}^{\infty}\left[1-\alpha+\frac{\alpha}{i !} \int_{0}^{\infty} \frac{e^{-y} y^{i}}{\left(1+(u / y)^{\beta}\right)^{m}} \mathrm{~d} y\right] \mathrm{d} u,
\end{aligned}
$$

where we substitute $u=m^{-1 / \beta} t$ and apply the beta function $B(x, y)=$ $\Gamma(x) \Gamma(y) / \Gamma(x+y)$ in the second equality.

It is known that $\alpha$-Ginibre point processes converge weakly to the homogeneous Poisson point process with the same intensity as $\alpha \rightarrow 0$ (see [13]). The following is an extension of Proposition 5 in [23], where the case of $F_{H}=\operatorname{Exp}(1)$ is considered.

Proposition 3.2. Let $C^{(\alpha-\mathrm{GPP})}\left(\beta, F_{H}\right)$ denote the asymptotic constant on the right-hand side of (B.16). Then, for any propagation effect distribution $F_{H}$ satisfying condition (A) of Theorem $\mathrm{L} . \mathrm{D}$,

$$
\lim _{\alpha \downarrow 0} C^{(\alpha-\mathrm{GPP})}\left(\beta, F_{H}\right)=\frac{\beta}{\pi} \sin \frac{\pi}{\beta} .
$$

Note that the right-hand side of (B.17) is just the asymptotic constant in Corollary 3.0 for the homogeneous Poisson-based model.

Pr o of. The proof essentially follows a similar line to that of Proposition 5 in [23]. Since the asymptotic constant (Z.T) in Theorem $[2$.$] does not depend on$ the intensity of the point process, we here choose $\lambda=\alpha / \pi$. Then, we can replace $Y_{i} \sim \operatorname{Gam}(i+1, \alpha)$ in Proposition B.] (ii) with $Y_{i} \sim \operatorname{Gam}(i+1,1)$. Clearly, the right-hand side of (B.16) is equal to

$$
C^{(\alpha-\mathrm{GPP})}\left(\beta, F_{H}\right)=\frac{\alpha \mathrm{E}\left(H_{1}{ }^{1 / \beta}\right)}{\Gamma(1+1 / \beta)} \int_{0}^{\infty} \prod_{i=1}^{\infty}\left\{1-\alpha \mathrm{E}\left[1-\mathcal{L}_{H}\left(\left(\frac{t}{Y_{i}}\right)^{\beta}\right)\right]\right\} \mathrm{d} t
$$

We use here the fact that, for any $\delta>0$, there exists an $x_{\delta} \in(0,1)$ such that $e^{-(1+\delta) x} \leqslant 1-x \leqslant e^{-x}$ for $x \in\left[0, x_{\delta}\right]$. Thus, for $\alpha \in\left(0, x_{\delta}\right]$, the integrand above has upper and lower bounds such as

$$
\begin{aligned}
& \text { (3.19) } \exp \left\{-(1+\delta) \alpha \sum_{i=1}^{\infty} \mathrm{E}\left[1-\mathcal{L}_{H}\left(\left(\frac{t}{Y_{i}}\right)^{\beta}\right)\right]\right\} \\
& \leqslant \prod_{i=1}^{\infty}\left\{1-\alpha \mathrm{E}\left[1-\mathcal{L}_{H}\left(\left(\frac{t}{Y_{i}}\right)^{\beta}\right)\right]\right\} \leqslant \exp \left\{-\alpha \sum_{i=1}^{\infty} \mathrm{E}\left[1-\mathcal{L}_{H}\left(\left(\frac{t}{Y_{i}}\right)^{\beta}\right)\right]\right\} .
\end{aligned}
$$


Here, applying the density function of $Y_{i} \sim \operatorname{Gam}(i+1,1), i \in \mathbb{N}$, we have

$$
\begin{aligned}
\sum_{i=1}^{\infty} \mathrm{E}\left[1-\mathcal{L}_{H}\left(\left(\frac{t}{Y_{i}}\right)^{\beta}\right)\right] & =\int_{0}^{\infty}\left(1-e^{-y}\right)\left[1-\mathcal{L}_{H}\left(\left(\frac{t}{y}\right)^{\beta}\right)\right] \mathrm{d} y \\
& =t \int_{0}^{\infty}\left(1-e^{-t u}\right)\left[1-\mathcal{L}_{H}\left(u^{-\beta}\right)\right] \mathrm{d} u
\end{aligned}
$$

where the last equality follows by substituting $u=y / t$. From the last expression above, we have, for any $t>0$,

$t \int_{0}^{\infty}\left[1-\mathcal{L}_{H}\left(u^{-\beta}\right)\right] \mathrm{d} u-1 \leqslant \sum_{i=1}^{\infty} \mathrm{E}\left[1-\mathcal{L}_{H}\left(\left(\frac{t}{Y_{i}}\right)^{\beta}\right)\right] \leqslant t \int_{0}^{\infty}\left[1-\mathcal{L}_{H}\left(u^{-\beta}\right)\right] \mathrm{d} u$,

and the common integral on both the sides reduces to

$$
\begin{aligned}
\int_{0}^{\infty}\left[1-\mathcal{L}_{H}\left(u^{-\beta}\right)\right] \mathrm{d} u & =\mathrm{E}\left[\int_{0}^{\infty}\left(1-e^{-H_{1} u^{-\beta}}\right) \mathrm{d} u\right] \\
& =\frac{\mathrm{E}\left(H_{1}{ }^{1 / \beta}\right)}{\beta} \int_{0}^{\infty}\left(1-e^{-v}\right) v^{-1-1 / \beta} \mathrm{d} v \\
& =\mathrm{E}\left(H_{1}{ }^{1 / \beta}\right) \Gamma\left(1-\frac{1}{\beta}\right),
\end{aligned}
$$

where the second equality follows by substituting $v=H_{1} u^{-\beta}$, and the last equality follows from the integration by parts. Hence, applying (3.19)-(B.22) to (B.J8) and using $\Gamma(x) \Gamma(1-x)=\pi \csc (\pi x)$ for $x \in(0,1)$, we obtain

$$
\frac{1}{1+\delta} \frac{\beta}{\pi} \sin \frac{\pi}{\beta} \leqslant C^{(\alpha-\mathrm{GPP})}\left(\beta, F_{H}\right) \leqslant e^{\alpha} \frac{\beta}{\pi} \sin \frac{\pi}{\beta} .
$$

The assertion follows as $\alpha \downarrow 0$ since $\delta$ is arbitrary.

3.3. A counterexample. Finally in this section, we give a simple counterexample that violates condition (B) of Theorem 2.$]$. Let $T$ denote a random variable with density function $f_{T}(t)=(a-1) t^{-a}, t \geqslant 1$, for $a \in(1,2)$. Note that $\mathrm{E} T=\infty$. Given a sample of $T$, we consider the mixed and randomly shifted lattice $\Phi=(\mathbb{Z} \times T \mathbb{Z})+U_{T}$, where $U_{T}$ denotes a uniformly distributed random variable on $[0,1] \times[0, T]$. The intensity $\lambda$ of $\Phi$ is then $\lambda=\mathrm{E}(1 / T)=(a-1) / a<\infty$. For any nonnegative and measurable function $g$, the definition of the Palm probability gives

$$
\mathrm{E}^{o} g(T)=\frac{1}{\lambda} \mathrm{E}(g(T) \Phi(I))=\frac{1}{\lambda} \mathrm{E}(g(T) \mathrm{E}(\Phi(I) \mid T))=\frac{1}{\lambda} \mathrm{E}\left(\frac{g(T)}{T}\right),
$$


where $I=[0,1]^{2}$. Hence, applying $R(o)^{2}=\left(1+T^{2}\right) / 4$ to the above, we have

$$
\mathrm{E}^{o}\left(R(o)^{2}\right)=\frac{1}{4 \lambda} \mathrm{E}\left(\frac{1}{T}+T\right)=\frac{1}{4 \lambda}(\lambda+\mathrm{E}(T))=\infty .
$$

\section{TAIL ASYMPTOTICS FOR BOUNDED PATH-LOSS MODELS}

In this section, we consider bounded and regularly varying path-loss functions. We assume that the distribution of propagation effects is light-tailed, and restrict ourselves to two cases of the point process $\Phi$; one is a homogeneous Poisson point process on $\mathbb{R}^{d}$ and the other is an $\alpha$-Ginibre point process on $\mathbb{C} \simeq \mathbb{R}^{2}$. In both the cases, we derive the same logarithmically asymptotic upper bound on the SIR tail distributions. Furthermore, when $\Phi$ is a homogeneous Poisson point process and the propagation effects are exponentially distributed, a logarithmically asymptotic lower bound with the same order as the upper bound is obtained. We first impose an assumption on the path-loss function $\ell$.

ASSUMPTION 4.1. $\ell$ is nonincreasing, bounded on $[0, \infty)$ and regularly varying at infinity with index $-d \beta, \beta>1$, in the sense that (see, e.g., [4], [28])

$$
\lim _{x \rightarrow \infty} \frac{\ell(t x)}{\ell(x)}=t^{-d \beta} \quad \text { for all } t>0 .
$$

In what follows, we suppose for simplicity that $\ell$ is bounded by one; that is, $\ell(r) \leqslant 1$ for $r \in[0, \infty)$. Let $g(s)=1 / \ell\left(s^{1 / d}\right), s \geqslant 0$. By Assumption 4.1] above, we see that the function $g$ is nondecreasing and regularly varying at infinity with index $\beta$. Thus, we can define an asymptotic inverse function $h$ of $g$ satisfying $g(h(z)) \sim h(g(z)) \sim z$ as $z \rightarrow \infty$ (see, e.g., [4], Section 1.5; [28], Chapter 1). The function $h$ is asymptotically unique and also regularly varying at infinity with index $1 / \beta$. For example, when $\ell(r)=\left(1+r^{d \beta}\right)^{-1}$, then $g(s)=$ $1 / \ell\left(s^{1 / d}\right)=1+s^{\beta}$, and we can take $h(z)=z^{1 / \beta}$. More generally, if $\ell(r)=$ $\left(1+r^{d \beta}[\log (1+r)]^{a}\right)^{-1}$ with $a \geqslant-d \beta$, then $g(s)=1+s^{\beta}\left[\log \left(1+s^{1 / d}\right)\right]^{a}$, and we can take $h(z)=z^{1 / \beta}(d \beta / \log z)^{a / \beta}$. The following theorem states that the SIR tail probability $\mathrm{P}\left(\operatorname{SIR}_{o}>\theta\right)$ is asymptotically bounded above by $e^{-\Theta(h(\theta))}$ as $\theta \rightarrow \infty$.

THEOREM 4.1. For the cellular network model described in Section $\square$ with the path-loss function satisfying Assumption 4.1 , we suppose that the distribution $F_{H}$ of the propagation effects $H_{i}, i \in \mathbb{N}$, satisfies the following:

(a) It is light-tailed; that is, there exists a (possibly infinite) $\zeta_{0}>0$ such that the moment generating function $\mathcal{M}_{H}(\zeta)=\mathrm{E} e^{\zeta H_{1}}$ is finite for $\zeta<\zeta_{0}$.

(b) The Laplace transform $\mathcal{L}_{H}$ satisfies $\log \mathcal{L}_{H}(s)=o\left(L_{1 / \beta}(s)\right)$ as $s \rightarrow \infty$ for any regularly varying function $L_{1 / \beta}$ with index $1 / \beta$. 
If $\Phi=\left\{X_{i}\right\}_{i \in \mathbb{N}}$ is a homogeneous Poisson point process on $\mathbb{R}^{d}$ with positive and finite intensity $\lambda$, then using the function $h$ defined above, we have

$$
\limsup _{\theta \rightarrow \infty} \frac{1}{h(\theta)} \log \mathrm{P}\left(\mathrm{SIR}_{o}>\theta\right) \leqslant-\pi_{d} \lambda \Gamma\left(1-\frac{1}{\beta}\right) \zeta_{0}^{1 / \beta} \mathrm{E}\left(H_{1}^{1 / \beta}\right),
$$

where $\zeta_{0}$ is the critical value for the existence of the moment generating function $\mathcal{M}_{H}$ of $H_{i}, i \in \mathbb{N}$. Moreover, if $d=2$ and $\Phi=\left\{X_{i}\right\}_{i \in \mathbb{N}}$ is an $\alpha$-Ginibre point process on $\mathbb{C} \simeq \mathbb{R}^{2}$, we have (4.1) as well.

Note that if $\zeta_{0}=\infty$, the SIR tail probability $\mathrm{P}\left(\mathrm{SIR}_{o}>\theta\right)$ decays faster than $e^{-\Theta(h(\theta))}$ as $\theta \rightarrow \infty$.

Pr o o f. Since $F_{H}$ is light-tailed, by Markov's inequality we obtain $\overline{F_{H}}(x)=$ $\mathrm{P}\left(e^{\zeta H_{i}}>e^{\zeta x}\right) \leqslant \mathcal{M}_{H}(\zeta) e^{-\zeta x}$ for $\zeta \in\left(0, \zeta_{0}\right)$. Thus, by $\ell(r) \leqslant 1$, (2.2) with replacing $\left|X_{i}\right|^{-d \beta}$ with $\ell\left(\left|X_{i}\right|\right)$ is bounded above as

$$
\begin{aligned}
\mathrm{P}\left(\mathrm{SIR}_{o}>\theta\right) & \leqslant \mathcal{M}_{H}(\zeta) \mathrm{E}\left[\prod_{i=2}^{\infty} \mathcal{L}_{H}\left(\zeta \theta \frac{\ell\left(\left|X_{i}\right|\right)}{\ell\left(\left|X_{1}\right|\right)}\right)\right] \\
& \leqslant \frac{\mathcal{M}_{H}(\zeta)}{\mathcal{L}_{H}(\zeta \theta)} \mathrm{E}\left[\prod_{i=1}^{\infty} \mathcal{L}_{H}\left(\zeta \theta \ell\left(\left|X_{i}\right|\right)\right)\right]
\end{aligned}
$$

Suppose that $\Phi=\left\{X_{i}\right\}_{i \in \mathbb{N}}$ is a homogeneous Poisson point process. For simplicity, we choose $\lambda=\pi_{d}{ }^{-1}$. Then, applying the probability generating functional (see, e.g., [10], Section 9.4) to the expectation above, we have

$$
\begin{aligned}
\mathrm{E}\left[\prod_{i=1}^{\infty} \mathcal{L}_{H}\left(\zeta \theta \ell\left(\left|X_{i}\right|\right)\right)\right] & =\exp \left\{-\frac{1}{\pi_{d}} \int_{\mathbb{R}^{d}}\left[1-\mathcal{L}_{H}(\zeta \theta \ell(|x|))\right] \mathrm{d} x\right\} \\
& =\exp \left\{-d \int_{0}^{\infty}\left[1-\mathcal{L}_{H}(\zeta \theta \ell(r))\right] r^{d-1} \mathrm{~d} r\right\} \\
& =\exp \left\{-\int_{0}^{\infty}\left[1-\mathcal{L}_{H}\left(\zeta \theta \ell\left(s^{1 / d}\right)\right)\right] \mathrm{d} s\right\},
\end{aligned}
$$

where the last equality follows by the substitution of $s=r^{d}$. Here, we set $\theta=$ $g(z)=1 / \ell\left(z^{1 / d}\right), z \geqslant 0$. Note that $\theta \rightarrow \infty$ as $z \rightarrow \infty$. Then, since $h(g(z)) \sim z$ as $z \rightarrow \infty$, we have

$$
\begin{aligned}
\lim _{\theta \rightarrow \infty} \frac{1}{h(\theta)} \int_{0}^{\infty}\left[1-\mathcal{L}_{H}\left(\zeta \theta \ell\left(s^{1 / d}\right)\right)\right] \mathrm{d} s & =\lim _{z \rightarrow \infty} \frac{1}{z} \int_{0}^{\infty}\left[1-\mathcal{L}_{H}\left(\zeta \frac{g(z)}{g(s)}\right)\right] \mathrm{d} s \\
& =\lim _{z \rightarrow \infty} \int_{0}^{\infty}\left[1-\mathcal{L}_{H}\left(\zeta \frac{g(z)}{g(z t)}\right)\right] \mathrm{d} t
\end{aligned}
$$

where $t=s / z$ is substituted in the second equality. We will confirm later whether 
the dominated convergence theorem is applicable in the last expression above and we now admit it. The regular variation of $g$ with index $\beta$ then yields

$$
\begin{aligned}
\lim _{z \rightarrow \infty} \int_{0}^{\infty}\left[1-\mathcal{L}_{H}\left(\zeta \frac{g(z)}{g(z t)}\right)\right] \mathrm{d} t & =\int_{0}^{\infty}\left[1-\mathcal{L}_{H}\left(\zeta t^{-\beta}\right)\right] \mathrm{d} t \\
& =\zeta^{1 / \beta} \mathrm{E}\left(H_{1}{ }^{1 / \beta}\right) \Gamma\left(1-\frac{1}{\beta}\right),
\end{aligned}
$$

where the second equality follows by a similar procedure to that for (B.22). Hence, applying (4.3)-(4.5) to (4.2) and taking $\zeta \rightarrow \zeta_{0}$, we obtain inequality (4.I) since $\log \mathcal{L}_{H}(\zeta \theta) / h(\theta) \rightarrow 0$ as $\theta \rightarrow \infty$ by condition (b) of the theorem.

Let us show that the dominated convergence theorem is applicable in (4.5)). Since $g$ is regularly varying with index $\beta$, we have $g(z)=z^{\beta} L_{0}(z)$ with a slowly varying function $L_{0}$, for which we can take a constant $B>0$ such that

$$
L_{0}(z)=\exp \left(\eta(z)+\int_{B}^{z} \frac{\epsilon(u)}{u} \mathrm{~d} u\right), \quad z \geqslant B,
$$

where $\eta(z)$ is bounded and converges to a constant as $z \rightarrow \infty$, and $\epsilon(u)$ is bounded and converges to zero as $u \rightarrow \infty$ (see, e.g., [4], Section 1.3, or [28], Chapter 1). We define constants $\eta^{*}$ and $\epsilon^{*}$ as

$$
\eta^{*}=\sup _{z \geqslant B}|\eta(z)|, \quad \epsilon^{*}=\sup _{z \geqslant B}|\epsilon(z)| .
$$

Note here that we can take $B$ large enough such that $\epsilon^{*}<\beta-1$. Then, for $z \geqslant B$ and $t \geqslant 1$, we have

$$
\frac{g(z)}{g(z t)} \leqslant t^{-\beta} e^{2 \eta^{*}} \exp \left(\epsilon^{*} \int_{z}^{z t} \frac{\mathrm{d} u}{u}\right)=e^{2 \eta^{*}} t^{-\left(\beta-\epsilon^{*}\right)},
$$

so that the integrand on the left-hand side of (4.5) satisfies

$$
1-\mathcal{L}_{H}\left(\zeta \frac{g(z)}{g(z t)}\right) \leqslant \mathbf{1}_{(0,1]}(t)+\left[1-\mathcal{L}_{H}\left(\zeta e^{2 \eta^{*}} t^{-\left(\beta-\epsilon^{*}\right)}\right)\right] \mathbf{1}_{(1, \infty)}(t) .
$$

As was the case with (3.22) (and (4.5)), the integral of the second term on the right-hand side above amounts to

$$
\begin{aligned}
\int_{1}^{\infty}\left[1-\mathcal{L}_{H}\left(\zeta e^{2 \eta^{*}} t^{-\left(\beta-\epsilon^{*}\right)}\right)\right] \mathrm{d} t & \leqslant \mathrm{E}\left[\int_{0}^{\infty}\left(1-e^{-\zeta e^{2 \eta^{*}} H_{1} t^{-\left(\beta-\epsilon^{*}\right)}}\right) \mathrm{d} t\right] \\
& =\left(\zeta e^{2 \eta^{*}}\right)^{1 /\left(\beta-\epsilon^{*}\right)} \mathrm{E}\left(H_{1}^{1 /\left(\beta-\epsilon^{*}\right)}\right) \Gamma\left(1-\frac{1}{\beta-\epsilon^{*}}\right) \\
& <\infty,
\end{aligned}
$$

and the dominated convergence theorem is applicable. 
Next, we show (4.J) when $d=2$ and $\Phi=\left\{X_{i}\right\}_{i \in \mathbb{N}}$ is an $\alpha$-Ginibre point process. Recall Proposition B.] (i), which states that $\left\{\left|X_{i}\right|^{2}\right\}_{i \in \mathbb{N}}$ has the same distribution as $\left\{\check{Y}_{i}\right\}_{i \in \mathbb{N}}$, and each $\check{Y}_{i}$ is extracted from $\left\{Y_{i}\right\}_{i \in \mathbb{N}}$ with probability $\alpha$ independently, where $Y_{i} \sim \operatorname{Gam}(i, \alpha), i \in \mathbb{N}$, are mutually independent. Applying this to (4.2), we have

$$
\mathrm{P}\left(\mathrm{SIR}_{o}>\theta\right) \leqslant \frac{\mathcal{M}_{H}(\zeta)}{\mathcal{L}_{H}(\zeta \theta)} \prod_{i=1}^{\infty} \mathrm{E}\left[1-\alpha+\alpha \mathcal{L}_{H}\left(\zeta \theta \ell\left(Y_{i}^{1 / 2}\right)\right)\right]
$$

so that, using $\log x \leqslant x-1$,

$$
\log \mathrm{P}\left(\mathrm{SIR}_{o}>\theta\right) \leqslant \log \frac{\mathcal{M}_{H}(\zeta)}{\mathcal{L}_{H}(\zeta \theta)}-\alpha \sum_{i=1}^{\infty} \mathrm{E}\left[1-\mathcal{L}_{H}\left(\zeta \theta \ell\left(Y_{i}^{1 / 2}\right)\right)\right] .
$$

Hence, applying the density function of $Y_{i} \sim \operatorname{Gam}(i, \alpha), i \in \mathbb{N}$, we obtain

$$
\begin{aligned}
\alpha \sum_{i=1}^{\infty} \mathrm{E}\left[1-\mathcal{L}_{H}(\zeta \theta \ell\right. & \left.\left.\left(Y_{i}^{1 / 2}\right)\right)\right] \\
& =\sum_{i=1}^{\infty} \int_{0}^{\infty} \frac{(y / \alpha)^{i-1} e^{-y / \alpha}}{(i-1) !}\left[1-\mathcal{L}_{H}\left(\zeta \theta \ell\left(y^{1 / 2}\right)\right)\right] \mathrm{d} y \\
& =\int_{0}^{\infty}\left[1-\mathcal{L}_{H}\left(\zeta \theta \ell\left(y^{1 / 2}\right)\right)\right] \mathrm{d} y,
\end{aligned}
$$

which is the same expression as the exponent in equality (4.3) and leads to the same result.

REMARK 4.1. We can see that many practical distributions satisfy condition (b) of Theorem A.1 Since $\mathcal{L}_{H}(s) \geqslant \mathrm{E}\left(e^{-s H_{1}} \mathbf{1}_{\left\{H_{1} \leqslant 1 / s\right\}}\right) \geqslant e^{-1} F_{H}(1 / s)$, we have $\left|\log \mathcal{L}_{H}(s)\right| \leqslant 1-\log F_{H}(1 / s)$. Thus, for example, if $F_{H}(x) \geqslant c x^{a}$ for $x \in[0, \epsilon]$ with $c>0, a \geqslant 0$ and $\epsilon>0$, then $\left|\log \mathcal{L}_{H}(s)\right|=O(\log s)$ as $s \rightarrow \infty$. On the other hand, a counterexample is such that there exists a constant $\epsilon>0$ with $F_{H}(\epsilon)=0$. Then, $\mathcal{L}_{H}(s) \leqslant e^{-\epsilon s}$, and we have $\left|\log \mathcal{L}_{H}(s)\right| \geqslant \epsilon s$.

REMARK 4.2. When $F_{H}=\operatorname{Exp}(1)$, we have $\mathcal{M}_{H}(s)=(1-s)^{-1}, s<1$. In this case, $\zeta_{0}=1$ and $\mathrm{E}\left(H_{1}{ }^{1 / \beta}\right)=\Gamma(1+1 / \beta)$ in Theorem 1.1 , so that, by $\Gamma(1+1 / \beta) \Gamma(1-1 / \beta)=(\pi / \beta) \csc (\pi / \beta)$, Theorem 4.1 reduces to

$$
\limsup _{\theta \rightarrow \infty} \frac{1}{h(\theta)} \log \mathrm{P}\left(\mathrm{SIR}_{o}>\theta\right) \leqslant-\frac{\pi}{\beta} \csc \frac{\pi}{\beta} .
$$

When $F_{H}=\operatorname{Exp}(1)$ and $\Phi$ is a homogeneous Poisson point process with positive and finite intensity, we can show that the tail distribution of the SIR has a logarithmically asymptotic lower bound which has the same order as the upper bound (4.7). 
PROPOSITION 4.1. For the cellular network model with the path-loss function satisfying Assumption 4.1 , when $F_{H}=\operatorname{Exp}(1)$ and $\Phi=\left\{X_{i}\right\}_{i \in \mathbb{N}}$ is a homogeneous Poisson point process on $\mathbb{R}^{d}$ with positive and finite intensity $\lambda$, we have

$$
\liminf _{\theta \rightarrow \infty} \frac{1}{h(\theta)} \log \mathrm{P}\left(\mathrm{SIR}_{o}>\theta\right) \geqslant-\pi_{d} \lambda \mathrm{E}\left[\ell\left(\left|X_{1}\right|\right)^{-1 / \beta}\right] \frac{\pi}{\beta} \csc \frac{\pi}{\beta} .
$$

Pr o o f. Applying $\overline{F_{H}}(x)=e^{-x}, x \geqslant 0$, and $\mathcal{L}_{H}(s)=(1+s)^{-1}, s \geqslant 0$, we rewrite (2.2) as

$$
\mathrm{P}\left(\mathrm{SIR}_{o}>\theta\right)=\mathrm{E}\left[\prod_{i=2}^{\infty}\left(1+\theta \frac{\ell\left(\left|X_{i}\right|\right)}{\ell\left(\left|X_{1}\right|\right)}\right)^{-1}\right] .
$$

Let the intensity of the Poisson point process be $\lambda=\pi_{d}^{-1}$. By concavity of logarithmic functions, Jensen's inequality yields

$$
\begin{aligned}
\log \mathrm{P}\left(\mathrm{SIR}_{o}>\theta\right) & \geqslant \mathrm{E}\left[\log \mathrm{E}\left[\prod_{i=2}^{\infty}\left(1+\theta \frac{\ell\left(\left|X_{i}\right|\right)}{\ell\left(\left|X_{1}\right|\right)}\right)^{-1}|| X_{1} \mid\right]\right] \\
& =-\frac{1}{\pi_{d}} \mathrm{E}\left[\int_{|x|>\left|X_{1}\right|}\left\{1-\left(1+\theta \frac{\ell(|x|)}{\ell\left(\left|X_{1}\right|\right)}\right)^{-1}\right\} \mathrm{d} x\right] \\
& =-\mathrm{E}\left[\int_{\left|X_{1}\right|^{d}}^{\infty}\left\{1-\left(1+\theta \frac{\ell\left(s^{1 / d}\right)}{\ell\left(\left|X_{1}\right|\right)}\right)^{-1}\right\} \mathrm{d} s\right],
\end{aligned}
$$

where we apply the probability generating functional to the conditional expectation given $\left|X_{1}\right|$ in the first equality and use a similar procedure to that for (4.3) in the last equality. Here, as in the proof of Theorem 4.1, we set $\theta=g(z)$. Then, since $h(g(z)) \sim z$ as $z \rightarrow \infty$, we have

$$
\begin{aligned}
\liminf _{\theta \rightarrow \infty} & \frac{1}{h(\theta)} \log \mathrm{P}\left(\mathrm{SIR}_{o}>\theta\right) \\
& \geqslant-\limsup _{z \rightarrow \infty} \frac{1}{z} \mathrm{E}\left[\int_{\left|X_{1}\right|^{d}}^{\infty}\left\{1-\left(1+\frac{1}{\ell\left(\left|X_{1}\right|\right)} \frac{g(z)}{g(s)}\right)^{-1}\right\} \mathrm{d} s\right] \\
& =-\limsup _{z \rightarrow \infty} \mathrm{E}\left[\int_{\left|X_{1}\right|^{d} / z}^{\infty}\left\{1-\left(1+\frac{1}{\ell\left(\left|X_{1}\right|\right)} \frac{g(z)}{g(z t)}\right)^{-1}\right\} \mathrm{d} t\right]
\end{aligned}
$$

where $t=s / z$ is substituted in the last equality. We will confirm later that the dominated convergence theorem is applicable to the above, and we have

$$
\lim _{z \rightarrow \infty} \mathrm{E}\left[\int_{\left|X_{1}\right|^{d} / z}^{\infty}\left\{1-\left(1+\frac{1}{\ell\left(\left|X_{1}\right|\right)} \frac{g(z)}{g(z t)}\right)^{-1}\right\} \mathrm{d} t\right]=\mathrm{E}\left[\int_{0}^{\infty} \frac{\mathrm{d} t}{1+\ell\left(\left|X_{1}\right|\right) t^{\beta}}\right] .
$$


Furthermore, substituting $u=\ell\left(\left|X_{1}\right|\right) t^{\beta}$, we obtain

$$
\int_{0}^{\infty} \frac{\mathrm{d} t}{1+\ell\left(\left|X_{1}\right|\right) t^{\beta}}=\frac{1}{\beta \ell\left(\left|X_{1}\right|\right)^{1 / \beta}} \int_{0}^{\infty} \frac{u^{1 / \beta-1}}{1+u} \mathrm{~d} u=\frac{1}{\ell\left(\left|X_{1}\right|\right)^{1 / \beta}} \frac{\pi}{\beta} \csc \frac{\pi}{\beta},
$$

which, together with (4.9) and (4.10), leads to (4.8)).

It remains to show whether the dominated convergence theorem is applicable in (4.10). Applying inequality (4.6), we have

$$
\begin{aligned}
\left\{1-\left(1+\frac{1}{\ell\left(\left|X_{1}\right|\right)}\right.\right. & \left.\left.\frac{g(z)}{g(z t)}\right)^{-1}\right\} \mathbf{1}_{\left\{\left|X_{1}\right| \leqslant(z t)^{1 / d}\right\}} \\
& \leqslant \mathbf{1}_{(0,1]}(t)+\left(1+e^{-2 \eta^{*}} \ell\left(\left|X_{1}\right|\right) t^{\beta-\epsilon^{*}}\right)^{-1} \mathbf{1}_{(1, \infty)}(t) .
\end{aligned}
$$

As was the case with (4.II), the integral of the second term on the right-hand side above amounts to

$$
\begin{aligned}
\int_{1}^{\infty} \frac{\mathrm{d} t}{1+e^{-2 \eta^{*}} \ell\left(\left|X_{1}\right|\right) t^{\beta-\epsilon^{*}}} & \leqslant \frac{e^{2 \eta^{*} /\left(\beta-\epsilon^{*}\right)}}{\left(\beta-\epsilon^{*}\right) \ell\left(\left|X_{1}\right|\right)^{1 /\left(\beta-\epsilon^{*}\right)}} \int_{0}^{\infty} \frac{u^{-1+1 /\left(\beta-\epsilon^{*}\right)}}{1+u} \mathrm{~d} u \\
& =\frac{e^{2 \eta^{*} /\left(\beta-\epsilon^{*}\right)}}{\ell\left(\left|X_{1}\right|\right)^{1 /\left(\beta-\epsilon^{*}\right)}} \frac{\pi}{\beta-\epsilon^{*}} \csc \frac{\pi}{\beta-\epsilon *} .
\end{aligned}
$$

It then suffices to show that $\mathrm{E}\left[\ell\left(\left|X_{1}\right|\right)^{-1 /\left(\beta-\epsilon^{*}\right)}\right]<\infty$. The regular variation of $\ell$ with index $-d \beta$ implies that $\ell(r)=r^{-d \beta} \widetilde{L}_{0}(r)$ with another slowly varying function $\widetilde{L}_{0}$, for which we can take a constant $\widetilde{B}>1$ such that

$$
\widetilde{L}_{0}(r)=\exp \left(\widetilde{\eta}(r)+\int_{\widetilde{B}}^{r} \frac{\widetilde{\epsilon}(t)}{t} \mathrm{~d} t\right), \quad r \geqslant \widetilde{B},
$$

where $\widetilde{\eta}(r)$ is bounded and converges to a constant as $r \rightarrow \infty$, and $\widetilde{\epsilon}(t)$ is bounded and converges to zero as $t \rightarrow \infty$. Define constants $\widetilde{\eta}_{\beta}$ and $\widetilde{\epsilon}_{\beta}$ as

$$
\widetilde{\eta}_{\beta}=\sup _{x \geqslant \widetilde{B}} \frac{|\widetilde{\eta}(x)|}{\beta-\epsilon^{*}}, \quad \widetilde{\epsilon}_{\beta}=\sup _{x \geqslant \widetilde{B}} \frac{|\widetilde{\epsilon}(x)|}{\beta-\epsilon^{*}} .
$$

Since $\ell$ is nonincreasing, we have

$$
\begin{aligned}
& \ell\left(\left|X_{1}\right|\right)^{-1 /\left(\beta-\epsilon^{*}\right)} \\
\leqslant & \ell(\widetilde{B})^{-1 /\left(\beta-\epsilon^{*}\right)} \mathbf{1}_{[0, \widetilde{B}]}\left(\left|X_{1}\right|\right)+\left|X_{1}\right|^{d} \widetilde{L}_{0}\left(\left|X_{1}\right|\right)^{-1 /\left(\beta-\epsilon^{*}\right)} \mathbf{1}_{(\widetilde{B}, \infty)}\left(\left|X_{1}\right|\right) \\
\leqslant & \ell(\widetilde{B})^{-1 /\left(\beta-\epsilon^{*}\right)}+e^{\widetilde{\eta}_{\beta}}\left|X_{1}\right|^{d+\widetilde{\epsilon}_{\beta}}
\end{aligned}
$$

which completes the proof since $\left|X_{1}\right|$ has any order of moments. 
REMARK 4.3. During the preparation of the first draft, the authors have found that the result in [15], Section IV-B, corresponds to our (4.7) and (4.8) for the homogeneous Poisson-based model with $d=2, F_{H}=\operatorname{Exp}(1)$ and $\ell(r)=$ $\left(1+r^{2 \beta}\right)^{-1}$. Here we deal with a much wider class of path-loss functions than that of power-law decaying functions.

REMARK 4.4. The results in this section hold when we relax the nonincreasing property of $\ell$ in Assumption 4.1 such that, for any finite $\widetilde{B}>0$, there exists an $\varepsilon_{\widetilde{B}}>0$ such that $\ell(r) \geqslant \varepsilon_{\widetilde{B}}$ for $r \in[0, \widetilde{B}]$, as we remain to assume the boundedness and regular variation. In this case, the proofs remain the same except for replacing $\ell(\widetilde{B})$ in $(4.12)$ with $\varepsilon_{\widetilde{B}}$.

\section{REFERENCES}

[1] J. G. Andrews, F. Baccelli, and R. K. Ganti, A tractable approach to coverage and rate in cellular networks, IEEE Trans. Commun. 59 (2011), pp. 3122-3134.

[2] S. Asmussen, J. L. Jensen, and L. Rojas-Nandayapa, On the Laplace transform of the lognormal distribution, Methodol. Comput. Appl. Probab. 18 (2016), pp. 441-458.

[3] F. B accelli and B. Błaszczyszy n, Stochastic Geometry and Wireless Networks. Volume I: Theory, Found. Trends Network. 3 (2009), pp. 249-449.

[4] N. H. Bingham, C. M. Goldie, and J. L. Teugels, Regular Variation, Cambridge University Press, Cambridge 1987.

[5] B. Błaszczyszyn, M. K. Karray, and H. P. Keeler, Wireless networks appear Poissonian due to strong shadowing, IEEE Trans. Wireless Commun. 14 (2015), pp. 4379-4390.

[6] B. Błaszczyszyn and D. Yogeshwaran, On comparison of clustering properties of point processes, Adv. in Appl. Probab. 46 (2014), pp. 1-20.

[7] B. Błaszczyszyn, D. Yogeshwaran, and J. E. Yukich, Limit theory for geometric statistics of clustering point processes, arXiv:1606.03988 [math.PR], 2016.

[8] P. Calka, The distributions of the smallest disks containing the Poisson-Voronoi typical cell and the Crofton cell in the plane, Adv. in Appl. Probab. 34 (2002), pp. 702-717.

[9] H. Chernoff, A measure of asymptotic efficiency for tests of a hypothesis based on the sum of observations, Ann. Math. Statist. 23 (1952), pp. 493-507.

[10] D. J. Daley and D. Vere-Jones, An Introduction to the Theory of Point Processes. Volume II: General Theory and Structure, second edition, Springer, 2008.

[11] S. G. Foss and S. A. Zuyev, On a Voronoi aggregative process related to a bivariate Poisson process, Adv. in Appl. Probab. 28 (1996), pp. 965-981.

[12] R. K. Ganti and M. Haenggi, Asymptotics and approximation of the SIR distribution in general cellular networks, IEEE Trans. Wireless Commun. 15 (2016), pp. 2130-2143.

[13] A. Goldman, The Palm measure and the Voronoi tessellation for the Ginibre process, Ann. Appl. Probab. 20 (2010), pp. 90-128.

[14] A. Guo and M. Haenggi, Asymptotic deployment gain: A simple approach to characterize the SINR distribution in general cellular networks, IEEE Trans. Commun. 63 (2015), pp. 962976.

[15] A. Guo, M. Haenggi, and R. K. Ganti, SIR asymptotics in general network models, arXiv:1611.04704 [cs.IT], 2016.

[16] M. Haenggi, The mean interference-to-signal ratio and its key role in cellular and amorphous networks, IEEE Wireless Commun. Lett. 3 (2014), pp. 597-600.

[17] W. Hoeffding, Probability inequalities for sums of bounded random variables, J. Amer. Statist. Assoc. 58 (1963), pp. 13-30. 
[18] J. B. Hough, M. Krishnapur, Y. Peres, and B. Virág, Zeros of Gaussian Analytic Functions and Determinantal Point Processes, Amer. Math. Soc., Providence, R.I., 2009.

[19] P. Keeler, N. Ross, A. Xia, and B. Błaszczyszyn, Stronger wireless signals appear more Poisson, IEEE Wireless Commun. Lett. 5 (2016), pp. 572-575.

[20] E. Kostlan, On the spectra of Gaussian matrices, Linear Algebra Appl. 162-164 (1992), pp. 385-388.

[21] J. Mercer, Functions of positive and negative type, and their connection with the theory of integral equations, Philos. Trans. A 209 (1909), pp. 415-446.

[22] N. Miyoshi and T. Shirai, A cellular network model with Ginibre configured base stations, Adv. in Appl. Probab. 46 (2014), pp. 832-845.

[23] N. Miyoshi and T. Shirai, Cellular networks with $\alpha$-Ginibre configurated base stations, in: The Impact of Applications on Mathematics: Proceedings of the Forum of Mathematics for Industry 2013, Springer, 2014, pp. 211-226.

[24] N. Miyoshi and T. Shirai, Downlink coverage probability in a cellular network with Ginibre deployed base stations and Nakagami-m fading channels, WiOpt 2015, pp. 483-489.

[25] N. Miyoshi and T. Shirai, A sufficient condition for tail asymptotics of SIR distribution in downlink cellular networks, WiOpt-SpaSWiN 2016, pp. 454-460.

[26] N. Miyoshi and T. Shirai, Spatial modeling and analysis of cellular networks using the Ginibre point process: A tutorial, IEICE Trans. Commun. E99-B (2016), pp. 2247-2255.

[27] H. Nagamatsu, N. Miyoshi, and T. Shirai, Padé approximation for coverage probability in cellular networks, WiOpt-SpaSWiN 2014, pp. 693-700.

[28] E. Seneta, Regularly Varying Functions, Springer, 1976.

[29] T. Shirai and Y. Takahashi, Random point fields associated with certain Fredholm determinants. I: Fermion, Poisson and boson processes, J. Funct. Anal. 205 (2003), pp. 414-463.

Naoto Miyoshi

Dept. of Mathematical and Computing Science

Tokyo Institute of Technology

2-12-1-W8-52 Ookayama

Tokyo 152-8552, Japan

E-mail: miyoshi@is.titech.ac.jp
Tomoyuki Shirai Institute of Mathematics for Industry Kyushu University 744 Motooka Fukuoka 819-0395, Japan E-mail: shirai@imi.kyushu-u.ac.jp

Received on 13.12.2016;

revised version on 15.3.2017 